\title{
THE CYCLIC HOMOLOGY OF ALGEBRAS WITH ADJOINED UNIT
}

\author{
GEORGE A. ELLIOTT, RYSZARD NEST, AND MIKAEL RØRDAM
}

(Communicated by Paul S. Muhly)

ABSTRACT. The effect on the cyclic homology of an algebra of adjoining a unit is calculated by means of an elementary analysis at the level of chains.

\section{INTRODUCTION}

Let $\mathscr{A}$ be an algebra over a field $k$ of characteristic zero. Let $\mathscr{A}^{\sim}$ denote the algebra over $k$ obtained by adjoining a unit to $\mathscr{A}$. One has a split short exact sequence

$$
0 \rightarrow \mathscr{A} \stackrel{i}{\rightarrow} \mathscr{A}^{\sim} \stackrel{p}{\rightleftarrows} k \rightarrow 0
$$

where the lifting $k \rightarrow \mathscr{A}^{\sim}$ maps a scalar into the corresponding multiple of the unit 1 in $\mathscr{A}^{\sim}$. By Propositions 4.1 and 4.2 of [2], (1) gives a split short exact sequence in cyclic homology,

$$
0 \rightarrow H_{n}^{\lambda}(\mathscr{A}) \stackrel{i_{*}}{\rightarrow} H_{n}^{\lambda}\left(\mathscr{A}^{\sim}\right) \stackrel{p_{*}}{\rightleftarrows} H_{n}^{\lambda}(k) \rightarrow 0,
$$

so that, in particular, one has

$$
H_{n}^{\lambda}\left(\mathscr{A}^{\sim}\right) \cong H_{n}^{\lambda}(\mathscr{A}) \oplus H_{n}^{\lambda}(k) .
$$

The purpose of this note is to give a description of this isomorphism at the level of chains. (We note that the arguments of [2] are valid also in the case of nonzero characteristic; we do not attempt to deal with this case.)

For each $x$ in $\mathscr{A}^{\sim}$ set $q(x)=x-p(x): 1$. Note that $q$ is a quasihomomorphism from $\mathscr{A}^{\sim}$ onto $\mathscr{A}$. We show that $q$ induces a map $q_{*}$ : $H_{n}^{\lambda}\left(\mathscr{A}^{\sim}\right) \rightarrow H_{n}^{\lambda}(\mathscr{A})$ and that $q_{*} \oplus p_{*}$ gives the isomorphism in (3).

\section{NOTATION AND A PRELIMINARY RESULT}

We give a brief introduction to the cyclic homology of A. Connes [1]. With $\mathscr{B}$ an algebra over a field $k$ of characteristic zero, let $\mathscr{B}^{\otimes(n+1)}$ denote the $(n+1)$-fold tensor product of $\mathscr{B}$ with itself. The Hochschild boundary operator

Received by the editors April 6, 1990.

1991 Mathematics Subject Classification. Primary 19D55, 18G99, 46M15, 46L80. 
$b: \mathscr{B}^{\otimes(n+1)} \rightarrow \mathscr{B}^{\otimes n}$ and the reduced boundary operator $b^{\prime}: \mathscr{B}^{\otimes(n+1)} \rightarrow \mathscr{B}^{\otimes n}$ are defined by

$$
\begin{array}{r}
b\left(a_{0} \otimes \cdots \otimes a_{n}\right)=\sum_{j=0}^{n-1}(-1)^{j} a_{0} \otimes \cdots \otimes a_{j} a_{j+1} \otimes \cdots \otimes a_{n} \\
+(-1)^{n} a_{n} a_{0} \otimes a_{1} \otimes \cdots \otimes a_{n-1}, \\
b^{\prime}\left(a_{0} \otimes \cdots \otimes a_{n}\right)=\sum_{j=0}^{n-1}(-1)^{j} a_{0} \otimes \cdots \otimes a_{j} a_{j+1} \otimes \cdots \otimes a_{n} .
\end{array}
$$

Define the cyclic permutation $\lambda: \mathscr{B}^{\otimes(n+1)} \rightarrow \mathscr{B}^{\otimes(n+1)}$ and the normalizing operator $A: \mathscr{B}^{\otimes(n+1)} \rightarrow \mathscr{B}^{\otimes(n+1)}$ by

$$
\begin{gathered}
\lambda\left(a_{0} \otimes \cdots \otimes a_{n}\right)=(-1)^{n} a_{n} \otimes a_{0} \otimes \cdots \otimes a_{n-1}, \\
A=1+\lambda+\lambda^{2}+\cdots+\lambda^{n} .
\end{gathered}
$$

Two $n$-chains (i.e. elements of $\left.\mathscr{B}^{\otimes(n+1)}\right) x$ and $y$ are said to be cyclically equal if $x-y \in \operatorname{Im}(1-\lambda)$, and we write $x \equiv y$. Equivalently, $x \equiv y$ if and only if $A x=A y$. The formula

$$
b^{\prime} A=A b
$$

implies that $b$ respects cyclic identifications. Let $Z_{n}^{\lambda}(\mathscr{B})$ be the set of all $n$ chains $x$ with $b x \equiv 0$, and let $B_{n}^{\lambda}(\mathscr{B})$ be the cyclic image of $\mathscr{B}^{\otimes(n+2)}$ under $b$, so that $x \in B_{n}^{\lambda}(\mathscr{B})$ if and only if $x \equiv b z$ for some $z$ in $\mathscr{B}^{\otimes(n+2)}$. The cyclic homology, $H_{n}^{\lambda}(\mathscr{B})$, of $\mathscr{B}$ is $Z_{n}^{\lambda}(\mathscr{B}) / B_{n}^{\lambda}(\mathscr{B})$.

A linear map $\phi: \mathscr{A} \rightarrow \mathscr{B}$ gives a linear map $\phi: \mathscr{A}^{\otimes(n+1)} \rightarrow \mathscr{B}^{\otimes(n+1)}$ defined by

$$
\phi\left(a_{0} \otimes \cdots \otimes a_{n}\right)=\phi\left(a_{0}\right) \otimes \cdots \otimes \phi\left(a_{n}\right), \quad a_{j} \in \mathscr{A} .
$$

If $\phi$ is an algebra homomorphism, then $\phi$ induces a homomorphism $\phi_{*}$ : $H_{n}^{\lambda}(\mathscr{A}) \rightarrow H_{n}^{\lambda}(\mathscr{B})$. If $\phi$ is a quasi-homomorphism, that is, a difference between two homomorphisms, then $\phi$ does not in general, at least by this specific process, induce a map on cyclic homology.

Proposition 1. Let $q(x)=x-p(x) \cdot 1, x \in \mathscr{A}^{\sim}$, be the canonical quasihomomorphism from $\mathscr{A}^{\sim}$ onto $\mathscr{A}$. Then $q$ induces a homomorphism $q_{*}$ : $H_{n}^{\lambda}\left(\mathscr{A}^{\sim}\right) \rightarrow H_{n}^{\lambda}(\mathscr{A})$, and $q_{*} i_{*}=\mathrm{id} \mid H_{n}^{\lambda}(\mathscr{A})$, where $i: \mathscr{A} \rightarrow \mathscr{A}^{\sim}$ is the inclusion mapping.

Proof. We show that

$$
b q x \equiv q b x, \quad x \in\left(\mathscr{A}^{\sim}\right)^{\otimes n+1},
$$

from which it will be clear that $q$ maps $Z_{n}^{\lambda}\left(\mathscr{A}^{\sim}\right)$ into $Z_{n}^{\lambda}(\mathscr{A})$ and $B_{n}^{\lambda}\left(\mathscr{A}^{\sim}\right)$ into $B_{n}^{\lambda}(\mathscr{A})$. Hence $q_{*}: H_{n}^{\lambda}\left(\mathscr{A}^{\sim}\right) \rightarrow H_{n}^{\lambda}(\mathscr{A})$ is well defined and it is obvious that $q_{*} i_{*}$ is the identity on $H_{n}^{\lambda}(\mathscr{A})$. 
To show (5), set $z=x-q x$ so that $q z=0$. Write $z$ as a linear combination of simple tensors $c_{0} \otimes \cdots \otimes c_{n}$ with either $c_{j} \in \mathscr{A}$ or $c_{j}=1$. We may assume that $c_{j}=1$ for at least one $j$ (otherwise $q\left(c_{0} \otimes \cdots \otimes c_{n}\right)=c_{0} \otimes \cdots \otimes c_{n}$ ). For any such $j$,

$$
\begin{aligned}
c_{0} \otimes \cdots \otimes c_{n} & \equiv(-1)^{n(n-j+1)} c_{j} \otimes \cdots \otimes c_{n} \otimes c_{0} \otimes \cdots \otimes c_{j-1} \\
& =(-1)^{n(n-j+1)} 1 \otimes c_{j+1} \otimes \cdots \otimes c_{n} \otimes c_{0} \otimes \cdots \otimes c_{j-1},
\end{aligned}
$$

and it follows that $z \equiv 1 \otimes z_{0}$ for some $z_{0}$ in $\left(\mathscr{A}^{\sim}\right)^{\otimes n}$. Thus

$$
\begin{aligned}
b x-b q x=b z & \equiv b\left(1 \otimes z_{0}\right)=(1-\lambda) z_{0}-1 \otimes b^{\prime} z_{0} \\
& \equiv-1 \otimes b^{\prime} z_{0},
\end{aligned}
$$

whence

$$
q b x-q b q x \equiv 0
$$

Since $b q x \in \mathscr{A}^{\otimes(n+1)}, q b q x=b q x$ and (5) follows from (6).

Note that Proposition 1 shows that the sequence (2) is exact at $H_{n}^{\lambda}(\mathscr{A})$. Exactness at $H_{n}^{\lambda}(k)$ follows from functoriality of cyclic homology. Also, we get a surjective homomorphism

$$
q_{*} \oplus p_{*}: H_{n}^{\lambda}\left(\mathscr{A}^{\sim}\right) \rightarrow H_{n}^{\lambda}(\mathscr{A}) \oplus H_{n}^{\lambda}(k) .
$$

In the next section we shall show that $q_{*} \oplus p_{*}$ is injective, and exactness of (2) at $H_{n}^{\lambda}\left(\mathscr{A}^{\sim}\right)$ will follow.

\section{THE MAIN RESULT}

For each $n$ define linear maps $F:\left(\mathscr{A}^{\sim}\right)^{\otimes(n+1)} \rightarrow k \otimes\left(\mathscr{A}^{\sim}\right)^{\otimes(n-1)} \otimes \mathscr{A}$ and $G: k \otimes\left(\mathscr{A}^{\sim}\right)^{\otimes n} \rightarrow\left(\mathscr{A}^{\sim}\right)^{\otimes n}$ by

$$
\begin{gathered}
F\left(a_{0} \otimes \cdots \otimes a_{n}\right)=p\left(a_{0}\right) \otimes a_{1} \otimes \cdots \otimes a_{n-1} \otimes q\left(a_{n}\right), \quad a_{j} \in \mathscr{A}^{\sim}, \\
G\left(1 \otimes a_{1} \otimes \cdots \otimes a_{n}\right)=a_{1} \otimes \cdots \otimes a_{n}, \quad a_{j} \in \mathscr{A}^{\sim} .
\end{gathered}
$$

Lemma 2. For each $x \in\left(\mathscr{A}^{\sim}\right)^{\otimes(n+1)}$,

$$
F b^{\prime} A x=b^{\prime} F A x-G F A x .
$$

Proof. By linearity of (8), it suffices to establish the lemma for a simple tensor $x=a_{0} \otimes \cdots \otimes a_{n}$ with either $a_{j} \in \mathscr{A}$ or $a_{j}=1$. In that case $A x=$ $\left(1+\lambda+\cdots+\lambda^{n}\right) x$ is a sum of $n+1$ tensors $c_{0} \otimes \cdots \otimes c_{n}$ with either $c_{j} \in \mathscr{A}$ or $c_{j}=1$. Now

$$
\left(F b^{\prime}-b^{\prime} F+G F\right)\left(c_{0} \otimes \cdots \otimes c_{n}\right)=0
$$

if either

(i) $c_{0} \in \mathscr{A}$,

(ii) $c_{n-1}=c_{n}=1$, or

(iii) $c_{0}=1$ and $c_{1}, c_{n} \in \mathscr{A}$; 
and, furthermore,

(iv) $\left(F b^{\prime}-b^{\prime} F+G F\right)\left(1 \otimes 1 \otimes c_{2} \otimes \cdots \otimes c_{n}\right)=1 \otimes c_{2} \otimes \cdots \otimes c_{n}, c_{n} \in \mathscr{A}$,

(v) $\left(F b^{\prime}-b^{\prime} F+G F\right)\left(1 \otimes c_{2} \otimes \cdots \otimes c_{n} \otimes 1\right)=(-1)^{n-1} 1 \otimes c_{2} \otimes \cdots \otimes c_{n}, c_{n} \in \mathscr{A}$.

To see (i) and (ii) note that $F b^{\prime}\left(c_{0} \otimes \cdots \otimes c_{n}\right)=F\left(c_{0} \otimes \cdots \otimes c_{n}\right)=0$ if $c_{0} \in \mathscr{A}$ or if $c_{n-1}=c_{n}=1$. To see (iii) assume $c_{0}=1$ and set $c=c_{1} \otimes \cdots \otimes c_{n}$. Then

$$
b^{\prime}(1 \otimes c)=c-1 \otimes b^{\prime} c \text {. }
$$

If $c_{1} \in \mathscr{A}$ and $c_{n} \in \mathscr{A}$, then $F c=0, F\left(1 \otimes b^{\prime} c\right)=1 \otimes b^{\prime} c$, and $F(1 \otimes c)=1 \otimes c$, so

$$
F b^{\prime}(1 \otimes c)=-1 \otimes b^{\prime} c=b^{\prime}(1 \otimes c)-c=b^{\prime} F(1 \otimes c)-G F(1 \otimes c) .
$$

To see (iv) and (v), set $c^{\prime}=c_{2} \otimes \cdots \otimes c_{n}$ and assume $c_{n} \in \mathscr{A}$. Then

$$
\begin{aligned}
& b^{\prime}\left(1 \otimes 1 \otimes c^{\prime}\right)=1 \otimes 1 \otimes b^{\prime} c, \\
& b^{\prime}\left(1 \otimes c^{\prime} \otimes 1\right)=c^{\prime} \otimes 1-1 \otimes b^{\prime} c^{\prime} \otimes 1+(-1)^{n-1} 1 \otimes c^{\prime} .
\end{aligned}
$$

Hence

$$
F b^{\prime}\left(1 \otimes 1 \otimes c^{\prime}\right)=1 \otimes 1 \otimes b^{\prime} c^{\prime}=b^{\prime} F\left(1 \otimes 1 \otimes c^{\prime}\right)
$$

and thus

$$
\left(F b^{\prime}-b^{\prime} F+G F\right)\left(1 \otimes 1 \otimes c^{\prime}\right)=G F\left(1 \otimes 1 \otimes c^{\prime}\right)=1 \otimes c^{\prime} .
$$

Since $F\left(c^{\prime} \otimes 1\right)=0=F\left(1 \otimes b^{\prime} c^{\prime} \otimes 1\right)$ so that

$$
F b^{\prime}\left(1 \otimes c^{\prime} \otimes 1\right)=(-1)^{n-1} 1 \otimes c^{\prime}
$$

we have

$$
\left(F b^{\prime}-b^{\prime} F+G F\right)\left(1 \otimes c^{\prime} \otimes 1\right)=(-1)^{n-1} 1 \otimes c^{\prime} .
$$

Finally, since

$$
\lambda\left(1 \otimes c_{2} \otimes \cdots \otimes c_{n} \otimes 1\right)=(-1)^{n} 1 \otimes 1 \otimes c_{2} \otimes \cdots \otimes c_{n},
$$

we see from (iv) and (v) that if $c_{n} \in \mathscr{A}$, then

$$
\begin{aligned}
\left(F b^{\prime}\right. & \left.-b^{\prime} F+G F\right)(1+\lambda)\left(1 \otimes c_{2} \otimes \cdots \otimes c_{n} \otimes 1\right) \\
& =(-1)^{n-1} 1 \otimes c_{2} \otimes \cdots \otimes c_{n}+(-1)^{n} 1 \otimes c_{2} \otimes \cdots \otimes c_{n}=0 .
\end{aligned}
$$

This, together with (i) to (iii), shows that $\left(F b^{\prime}-b^{\prime} F+G F\right) A x=0$.

Define $P:\left(\mathscr{A}^{\sim}\right)^{\otimes(n+1)} \rightarrow\left(\mathscr{A}^{\sim}\right)^{\otimes(n+1)}$ to be

$$
P=\sum_{r=0}^{n} \lambda^{r} F \lambda^{-r}
$$

If $c=c_{0} \otimes \cdots \otimes c_{n}$ is a simple tensor in $\left(\mathscr{A}^{\sim}\right)^{\otimes(n+1)}$ with either $c_{j} \in \mathscr{A}$ or $c_{j}=1$, then either $F c=c$ or $F c=0$, and, further, either $F \lambda^{-r} c=\lambda^{-r} c$ or $F \lambda^{-r} c=0$. Hence $P c=l c$ for some integer $l \geq 0$. (This integer counts the number of groups of consecutive 1's in $c_{0} \otimes \cdots \otimes c_{n}$ if $c_{0}$ is thought of 
as following $c_{n}$, and provided that not all $c_{j}$ are 1.) We have $P c=0$ if and only if all $c_{j} \in \mathscr{A}$ or all $c_{j}=1$, equivalently, if and only if either $q(c)=c$ or $p(c)=c$. More simply put, $P c=0$ if and only if $p c+q c=c$.

Upon writing any chain $x$ in $\left(\mathscr{A}^{\sim}\right)^{\otimes(n+1)}$ as a linear combination of simple tensors, we see that $P$ has spectrum contained in $\{0,1, \ldots, n\}$, that the eigenvectors of $P \operatorname{span}\left(\mathscr{A}^{\sim}\right)^{\otimes(n+1)}$ and that

$$
P x=0 \Leftrightarrow p x+q x=x .
$$

Thus we may define a partial inverse $Q$ to $P$ by

$$
Q x= \begin{cases}0 & \text { if } P x=0, \\ l^{-1} x & \text { if } P x=l x, l \neq 0\end{cases}
$$

and then

$$
Q P x=x \Leftrightarrow p x=q x=0 .
$$

Lemma 3. If $x \in\left(\mathscr{A}^{\sim}\right)^{\otimes(n+1)}$ and $p x=q x=0$, then $x$ is cyclically equal to QFAX.

Proof. We must show that $A x=A Q F A x$. Note first that $\lambda$ commutes with $P$ and hence with $Q$. Thus

$$
\begin{aligned}
A Q F A x & =\sum_{r=0}^{n} \sum_{s=0}^{n} \lambda^{r} Q F \lambda^{s} x=\sum_{r=0}^{n} \sum_{s=0}^{n} Q \lambda^{r} F \lambda^{s} x \\
& =\sum_{r^{\prime}=0}^{n} \sum_{s^{\prime}=0}^{n} Q \lambda^{r^{\prime}} \lambda^{s^{\prime}} F \lambda^{-s^{\prime}} x=Q A P x \\
& =A Q P x=A x .
\end{aligned}
$$

Define $b_{0}: k \otimes\left(\mathscr{A}^{\sim}\right)^{\otimes n} \rightarrow k \otimes\left(\mathscr{A}^{\sim}\right)^{\otimes(n+1)}$ by

$$
b_{0}(1 \otimes a)=-1 \otimes b^{\prime} a, \quad a \in\left(\mathscr{A}^{\sim}\right)^{\otimes n} .
$$

For each $y$ in $k \otimes\left(\mathscr{A}^{\sim}\right)^{\otimes n}$, the following formulas are easily verified:

$$
\begin{aligned}
b_{0} y & =b^{\prime} y-G y, \\
b(1 \otimes y) & =-1 \otimes b_{0} y-\lambda y .
\end{aligned}
$$

Lemma 4. For each $z$ in the image of $F$ we have

$$
b_{0} P z=P b_{0} z \text { and } b_{0} Q z=Q b_{0} z \text {. }
$$

Proof. From the definition of $Q$ it follows that each operator that commutes with $P$ will also commute with $Q$, and so it suffices to show that $b_{0} P z=$ $P b_{0} z$. Secondly, it will suffice to show $b_{0} P z=P b_{0} z$ for each simple tensor $z=c_{0} \otimes \cdots \otimes c_{n}$, in the image of $F$, with either $c_{j} \in \mathscr{A}$ or $c_{j}=1$. We must have $c_{0}=1$ and $c_{n} \in \mathscr{A}$, and so we may write $z$ as

$$
z=1 \otimes c^{(1)} \otimes 1 \otimes c^{(2)} \otimes \cdots \otimes 1 \otimes c^{(l)},
$$


where

$$
c^{(j)}=\underbrace{1 \otimes \cdots \otimes 1}_{r_{j}} \otimes c_{r_{j}+1}^{(j)} \otimes \cdots \otimes c_{s_{j}}^{(j)}, \quad j=1, \ldots, l,
$$

$c_{r}^{(j)} \in \mathscr{A}$ for $r>r_{j}$, and $s_{j}>r_{j} \geq 0$ for $j=1, \ldots, l$. It is easy to see that $P z=l z$. Moreover,

$$
b_{0} z=-\sum_{j=1}^{l} \pm 1 \otimes c^{(1)} \otimes \cdots \otimes 1 \otimes b^{\prime} c^{(j)} \otimes \cdots \otimes 1 \otimes c^{(l)} .
$$

From the structure of $c^{(j)}$ we see that each term on the right hand side of (14) has exactly $l$ groups of consecutive l's (in the sense described below the proof of Lemma 2), and we conclude that $P b_{0} z=l b_{0} z$. Hence $b_{0} P z=l b_{0} z=P b_{0} z$ as desired.

Lemma 5. If $x \in Z_{n}^{\lambda}\left(\mathscr{A}^{\sim}\right)$ and $p(x)=q(x)=0$, then

$$
x \equiv-b(1 \otimes Q F A x),
$$

so $x \in B_{n}^{\lambda}\left(\mathscr{A}^{\sim}\right)$.

Proof. By (13), Lemma 4, (12), Lemma 2, (4), and the hypothesis $A b x=0$, we have

$$
\begin{aligned}
-b(1 \otimes Q F A x) & =1 \otimes b_{0} Q F A x+\lambda Q F A x \\
& =1 \otimes Q b^{\prime} F A x-1 \otimes Q G F A x+\lambda Q F A x \\
& =1 \otimes Q F b^{\prime} A x+\lambda Q F A x \\
& =1 \otimes Q F A b x+\lambda Q F A x \\
& =\lambda Q F A x .
\end{aligned}
$$

By Lemma 3, we have

$$
\lambda Q F A x \equiv Q F A x \equiv x
$$

Theorem 6. The map

$$
q_{*} \oplus p_{*}: H_{n}^{\lambda}\left(\mathscr{A}^{\sim}\right) \rightarrow H_{n}^{\lambda}(\mathscr{A}) \oplus H_{n}^{\lambda}(k)
$$

is an isomorphism.

Proof. In Proposition 1 we saw that $q_{*} \oplus p_{*}$ is well defined and surjective. To see that $q_{*} \oplus p_{*}$ is injective, let $x \in Z_{n}^{\lambda}\left(\mathscr{A}^{\sim}\right)$ and set $z=x-p(x)-q(x)$. Since $p(x) \in Z_{n}^{\lambda}(k)$ and $q(x) \in Z_{n}^{\lambda}(\mathscr{A})$ (by Proposition 1) we have $z \in Z_{n}^{\lambda}\left(\mathscr{A}^{\sim}\right.$ ) and $p(z)=q(z)=0$. From Lemma 5 it follows that $z \in B_{n}^{\lambda}\left(\mathscr{A}^{\sim}\right)$, that is, $[z]=0$, so if $p_{*}[x]=q_{*}[x]=0$, then $[x]=0$. 
As noted in the remarks after Proposition 1, Theorem 6 implies

Corollary 7 [2]. The sequence

$$
0 \rightarrow H_{n}^{\lambda}(\mathscr{A}) \rightarrow H_{n}^{\lambda}\left(\mathscr{A}^{\sim}\right) \leftrightarrows H_{n}^{\lambda}(k) \rightarrow 0
$$

is exact.

We remark that Corollary 7 can be used to extend Connes's Chern character ch: $K_{0}(\mathscr{A}) \rightarrow H_{2 n}^{\lambda}(\mathscr{A})$ for unital algebras to include nonunital algebras. Indeed, we have a commutative diagram with exact rows

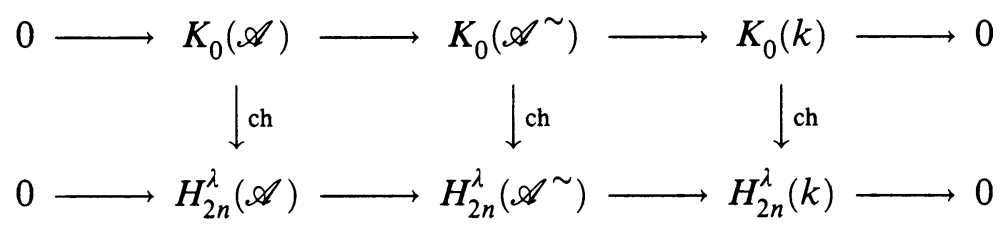

that induces ch: $K_{0}(\mathscr{A}) \rightarrow H_{2 n}^{\lambda}(\mathscr{A})$. Note that Connes's construction of the Chern character in the odd dimensional case is already defined for nonunital algebras.

\section{ACKNOWLEDGMENTS}

This work was supported by grants from the Natural Sciences and Engineering Research Council of Canada and the Danish Natural Science Research Council. The first author was on leave of absence from the University of Copenhagen.

\section{BIBLIOGRAPHY}

1. A. Connes, Non-commutative differential geometry, Parts I and II, Inst. Hautes Études Sci. Publ. Math. 62 (1985), 257-360.

2. J.-L. Loday and D. Quillen, Cyclic homology and the Lie algebra homology of matrices, Comment. Math. Helv. 59 (1984), 565-591.

Department of Mathematics, University of Toronto, Toronto, Canada M5S 1A1

Current address, Ryszard Nest: Mathematics Institute, University of Copenhagen, Copenhagen, Denmark mark

Current address, Mikael Rørdam: Mathematics Institute, University of Odense, Odense, Den- 